\title{
Relation between Morphology and Chiroptical Properties in Chiral Conducting Polymer Films: A Case Study in Chiral PEDOT
}

\author{
Dana Amsallem, Anjan Bedi, Francesco Tassinari, and Ori Gidron* \\ Cite This: Macromolecules 2020, 53, 9521-9528 \\ Read Online
}

ABSTRACT: The electronic properties of conducting polymers are influenced by their micro- and macrostructural orders, which can be tailored by substituent modification. However, while the effect of substituents on conducting polymers is extensively investigated, chiral substituents are far less studied. Furthermore, many chiral conducting polymers have regioirregular structures, which result in polymer films with inferior properties. In this work, we apply electronic circular dichroism (ECD) spectroscopy to

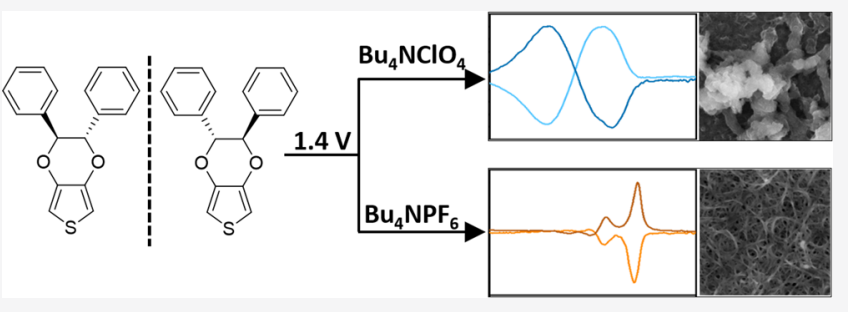
study the morphological changes to the chiral polymers under different polymerization conditions. For this purpose, we investigated 3,4-ethylenedioxythiophene (EDOT) derivatives having two stereogenic centers on each monomer and bearing methyl or phenyl side groups (dimethyl-EDOT and diphenyl-EDOT, respectively). Polymerizing the enantiomerically pure monomers produces regioregular and stereoregular dimethyl-PEDOT and diphenyl-PEDOT, respectively. The effect of the electrolyte and solvent on polymer film morphology was studied using scanning electron microscopy (SEM) and ECD, showing a correlation between the polymer's morphology and the chiroptical properties of its films. We found that, for diphenyl-PEDOT, the combination of perchlorate anion electrolyte and acetonitrile solvent resulted in a unique morphology characterized by significant intermolecular interactions. These interactions were clearly observable in the ECD spectra in the form of exciton couplings, whose presence was supported by TD-DFT calculations. A small enantiomeric excess was sufficient to induce very intense ECD signals, demonstrating chiral amplification in electropolymerized films.

\section{INTRODUCTION}

Chiral conducting polymers have numerous applications, including chiral sensors and catalysts, chemical separation materials, and spintronic devices. ${ }^{1-5}$ Among conducting polymers, poly(3,4-ethylenedioxythiophene) (PEDOT) is one of the most extensively studied because of the stability of its doped and undoped states, the ease with which it is structurally modified, and its processability. ${ }^{6-8}$ The disubstitution of the monomer at both $\beta$-positions reduces its tendency to engage in cross-linking during the polymerization process. potentials can be tailored via substitution with additional side groups. ${ }^{10}$ The addition of chiral substituents can result in interesting features. ${ }^{2,10}$ For example, polythiophenes bearing chiral side groups were recently applied as spin filters ${ }^{4}$ and magneto-optical materials. ${ }^{11}$

Chirality can be probed using electronic circular dichroism (ECD), which also provides information about supramolecular features (e.g., exciton coupling) in a less ambiguous manner compared with absorption spectroscopy. ${ }^{10}$ The relation between polymer film morphology and ECD signal was previously studied by Akagi's group for chemically prepared PEDOT polymer films with chiral spacers. They observed that the chiroptical properties weakened upon oxidation, which was ascribed to the distancing of helically arranged polymer chains by the inter-relation of counteranions. ${ }^{12,13}$ Goto has studied the optical properties of chiral PEDOT film prepared in cholesteric liquid crystals. ${ }^{14}$ In contrast, little is known about the relation between chiroptical properties and morphology in films electrochemically prepared from chiral monomers. Bäuerle's group ${ }^{15}$ used transetherification followed by electropolymerization to synthesize a series of chiral EDOT monomers from 3,4-dimethoxy-thiophene and chiral diols. However, neither the chiroptical properties nor the morphology of these polymers was investigated. Different functional groups directly affect the morphology of PEDOT films. ${ }^{16,17}$ In addition, the use of different electrochemical conditions is known to affect the morphology of conducting polymers and hence influence their physical and electrochemical properties, such as electrical conductivity, electrochromism, optical properties, and stability. ${ }^{18-20}$ However, it is not always trivial to understand these morphological differences at the supramolecular level. ${ }^{21-23} \mathrm{We}$ were therefore interested in

Received: July 25, 2020

Revised: October 7, 2020

Published: October 22, 2020 
monitoring the change in morphology of chiral polymer films using ECD.

In this work, we investigate the chiroptical properties of chiral PEDOT films electropolymerized under various conditions. To this end, we introduce monomers diphenylEDOT (1, Chart 1) and dimethyl-EDOT (2, Chart 1) and

Chart 1. 3,4-Ethylenedioxythiophene (EDOT) Derivatives Disubstituted with Phenyl Rings (S,S-1, meso-1, and R,R-1) or with Methyl Groups (S,S-2 and $R, R-2)$, where 1 is Diphenyl-EDOT and 2 is Dimethyl-EDOT

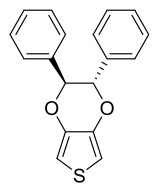

$S, S-1$

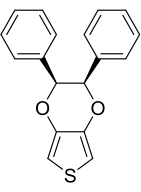

meso-1

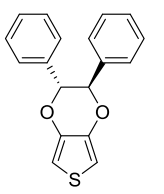

$R, R-1$

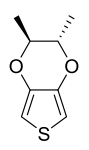

$S, S-2 \quad R, R-2$ compare the film morphologies of their polymers P1 and P2 using ECD together with traditional techniques (scanning electron microscopy (SEM), UV-vis, contact angle, and electrochemistry). We find that the ECD spectra of films electropolymerized under different conditions correlate well with their SEM morphologies, and provide important insights into the arrangement of the polymers at the supramolecular level. Control experiments with polymers synthesized from achiral EDOT and linear dichroism (LD) techniques indicate that the chirality stems from both interchain and intrachain helical arrangements, and density functional theory (DFT) calculations support this finding. Unexpectedly, films of mesodiphenyl-PEDOT (meso-P1) exhibit strong optical activity, with further experimentation indicating that this stems from a small enantiomeric excess, which amplifies to become a very intense signal. Electropolymerization of films containing various degrees of enantiomeric excess indicates that the majority rule, whereby the enantiomer that forms the majority of the mixture determines the chirality of the overall assembly process, ${ }^{24}$ applies not only to chemical polymerization but also the electrodeposition of conducting polymers.

\section{EXPERIMENTAL SECTION}

Reagent grade chemicals were used without further purification. Flash chromatography (FC) was performed using a CombiFlash EZ-Prep equipped with $\mathrm{SiO}_{2}$ columns. UV-vis absorption spectra were recorded with an Agilent Cary-5000 spectrophotometer. The spectra were measured using a quartz cuvette $(1 \mathrm{~cm})$ at $25{ }^{\circ} \mathrm{C}$. The absorption wavelengths are reported in $\mathrm{nm}$ with the extinction coefficient $\varepsilon\left(\mathrm{M}^{-1} \mathrm{~cm}^{-1}\right)$ given in brackets. Electronic circular dichroism (ECD) spectra were recorded on a MOS-500 spectrophotometer (BioLogic Science Instruments). For electrochemical measurements, acetonitrile was used as the solvent. Electrochemical solutions contained one of the following electrolytes at a concentration of $0.1 \mathrm{M}$ : tetra- $n$-butylammonium perchlorate $\left(\mathrm{Bu}_{4} \mathrm{NClO}_{4}\right)$, tetra- $n$-butylammonium tetrafluoroborate $\left(\mathrm{Bu}_{4} \mathrm{NBF}_{4}\right)$, tetra- $n$-butylammonium trifluoromethylsulfonate $\left(\mathrm{Bu}_{4} \mathrm{NCF}_{3} \mathrm{SO}_{3}\right)$, tetra- $n$-butylammonium hexafluorophosphate $\left(\mathrm{Bu}_{4} \mathrm{NPF}_{6}\right)$, or lithium perchlorate $\left(\mathrm{LiClO}_{4}\right) . \mathrm{Ag} / \mathrm{AgCl}$ was used as a (pseudo) reference electrode. A ferrocene/ferrocenium redox couple $\left(\mathrm{Fc} / \mathrm{Fc}^{+}=0.34 \mathrm{~V}\right.$ vs $\mathrm{SCE}$ ) was used as an internal reference for all measurements. The $\mathrm{Ag} /$ $\mathrm{AgCl}$ electrode was coated by dipping a silver wire in an aqueous solution of $\mathrm{FeCl}_{3}$ and $\mathrm{HCl}$. Platinum-wire electrodes were applied as the counter electrode. Two types of working electrodes were used: platinum-disk and glass coated with indium tin oxide (ITO) on one surface. ITO working electrodes $\left(7 \times 5 \times 0.7 \mathrm{~mm}^{3}\right)$ were purchased from Delta Technologies. $\mathrm{Ti}, \mathrm{Ni}$, and $\mathrm{Au}$ were evaporated onto a silicon wafer (thickness, $100 \mathrm{~mm}$; orientated $\langle 100\rangle \pm 0.5$; doped with boron, having resistance $\leq 0.0015 \Omega / \mathrm{cm}$ ) to form layers of thickness of 3,50 , and $10 \mathrm{~nm}$, respectively. The wafer was cut to form $1 \times 2 \mathrm{~cm}^{2}$ working electrodes. All electrochemical measurements were performed under a dry nitrogen atmosphere and externally calibrated against the $E^{1 / 2}$ of the $\mathrm{Fc} / \mathrm{Fc}^{+}$redox couple. Anhydrous acetonitrile was purchased from Sigma-Aldrich and used as received. Scanning electron microscopy (SEM) images were taken at the Hebrew University Center for Nanoscience and Nanotechnology using an XHR SEM FEI Magellan 400L (the Netherlands) instrument. The working conditions were $2 \mathrm{kV}$ and $0.2 \mathrm{nA}$. Contact angle goniometry measurements were carried out using triply distilled water (TDW) droplets and a Theta Lite optical tensiometer controlled by OneAttension software (Biolin Scientific).

\section{RESULTS AND DISCUSSION}

Preparation and Electrochemical Properties of Chiral Polymer Films. Derivatives $\mathbf{1}$ and $\mathbf{2}$ were synthesized via acidcatalyzed transetherification using the method reported by Bäuerle. ${ }^{15}$ The reaction between 3,4-dimethoxythiophene and chiral glycols produces an EDOT monomer disubstituted at the ethylene bridge (Scheme 1). Since positions 3 and 4 in the thiophene ring are substituted with the same group, the resultant PEDOT is regioregular. In the case of enantiomerically pure monomers, the resultant polymer is stereoregular as well, whereas in the case of a meso monomer, the resultant polymer is regioregular but not stereoregular.

Films of diphenyl-PEDOT and dimethyl-PEDOT (P1 and P2, respectively) were electrochemically prepared on ITO and Pt working electrodes by applying a constant potential of $1.4 \mathrm{~V}$.

Scheme 1. Acid-Catalyzed Transetherification Followed by Electrodeposition ${ }^{a}$

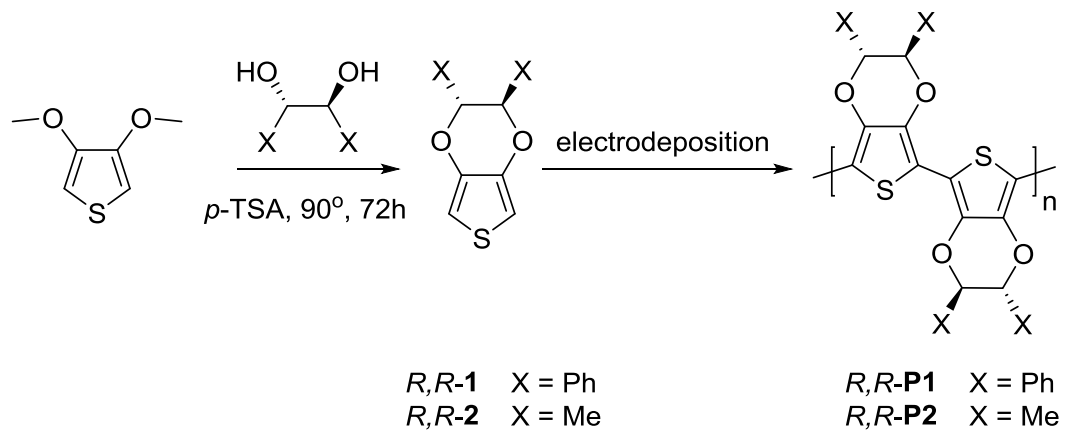

${ }^{a}$ Reaction of 3,4-dimethoxythiophene with chiral glycols produces an EDOT monomer disubstituted at the ethylene bridge. p-TSA, paratoluenesulfonic acid. 
The UV-vis absorption spectra for neutral films of P1 and P2 display the expected $\pi-\pi^{*}$ transition at ca. $600-700 \mathrm{~nm}^{25}$ Upon oxidation, the appearance of a polaron is observed at ca. $1000 \mathrm{~nm}$ with a maximum intensity of around $0.4 \mathrm{~V}$. Further oxidation results in the evolution of the absorption band at ca. 1500-2200 nm, corresponding to a bipolaron species (Figure S22, see SI).

The ECD spectra of enantiomerically pure $R, R$ polymers (dashed lines) and $S, S$ polymers (solid lines) are mirror images of each other, as expected, for both P1 (Figure 1a) and P2
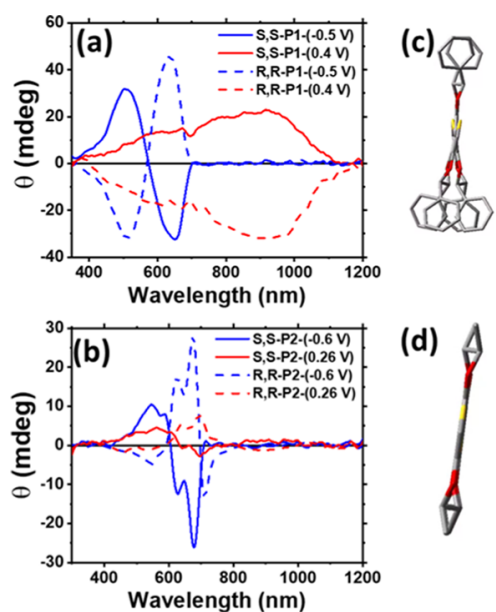

(d)



Figure 1. ECD spectroelectrochemistry of films containing (a) S,S-P1 (solid line) and R,R-P1 (dashed line) in the neutral (blue) and semioxidized (red) states and (b) S,S-P2 (solid line) and R,R-P2 (dashed line) in the neutral (blue) and semioxidized (red) states, in acetonitrile with $0.1 \mathrm{M} \mathrm{Bu}_{4} \mathrm{NClO}_{4}$. Recorded on ITO as the working electrode and $\mathrm{Ag} / \mathrm{AgCl}$ reference electrode $\left(\mathrm{Fc} / \mathrm{Fc}^{+}=0.34 \mathrm{~V}\right.$ vs SCE under these conditions). Optimized structures (DFT/B3LYP/6$31 \mathrm{G}(\mathrm{d}))$ of (c) ter-1 and (d) ter-2.

(Figure 2a) in their neutral and oxidized states. This indicates that the configuration of the phenyl or methyl side groups determines the direction of the helicity established during polymerization. The bisignate peak observed for neutral P1 (blue) indicates that the polymer chains lie in close proximity to each other and in a tilted orientation. ${ }^{10,26}$ According to the bisignate Cotton effect, the characteristics of the longer wavelength band are dictated by the directionality of the assembly. Specifically, two $R$ centers will lead to a positive lowenergy band, corresponding to clockwise $(P)$ helical assembly, whereas two $S$ centers will lead to a negative low-energy band and a counter-clockwise $(M)$ helical assembly. ${ }^{1,2}$ Upon oxidation, the bisignate peak is replaced by a broad ECD monosignate peak (red), with no indication of the exciton coupling observed for the neutral film. This likely results from the polymer chains separating following ion interchelation upon oxidation, as was previously reported. ${ }^{12,14}$ The ECD spectrum for P2 (Figure 1b) is substantially different from that of P1 (Figure 1a): whereas exciton coupling can be observed in the neutral state (blue), no peak is observed for the polaron in the semioxidized state (red).

To understand the reason for the difference between methyl and phenyl substituents, the trimers of $\mathbf{1}$ and $\mathbf{2}$ (ter-1 and ter-2, respectively) were calculated at the DFT/B3LYP/6-31G(d) level. As can be observed in Figure 1c, the backbone of ter-1 is twisted (dihedral angle of $171^{\circ}$ ), whereas the backbone of ter2, which is substituted with the less sterically hindered methyl groups, is planar (dihedral angle of $179^{\circ}$; Figure 1d). Thus, upon interchelation of the anions, the chains in both P1 and P2 polymer films move further apart, such that the interchain exciton coupling becomes negligible. Thus, the polaron ECD signal for P1 results from the intrachain helicity, while the absence of such a signal in $\mathbf{P 2}$ indicates the absence of intrachain helicity.

Morphology and Optical Activity. To study the scope for using ECD as a tool to assess film morphology, 1, 2, and EDOT were electropolymerized using different supporting electrolytes $\left(\mathrm{Bu}_{4} \mathrm{NClO}_{4}, \mathrm{LiClO}_{4}, \mathrm{Bu}_{4} \mathrm{NCF}_{3} \mathrm{SO}_{3}, \mathrm{Bu}_{4} \mathrm{NPF}_{6}\right.$, or $\mathrm{Bu}_{4} \mathrm{NBF}_{4}$ ) and solvents (acetonitrile or propylene carbonate) under chronoamperometric conditions. The ECD spectra were analyzed together with SEM and contact angle (CA) measurements.

Figure 2 displays the ECD spectra and SEM images for $R, R$ $\mathbf{P 1}$ and S,S-P1 polymerized using different electrolytes in acetonitrile. The $R, R$ - and $S, S$-enantiomers display mirror image spectra, which indicates that the source of the observed
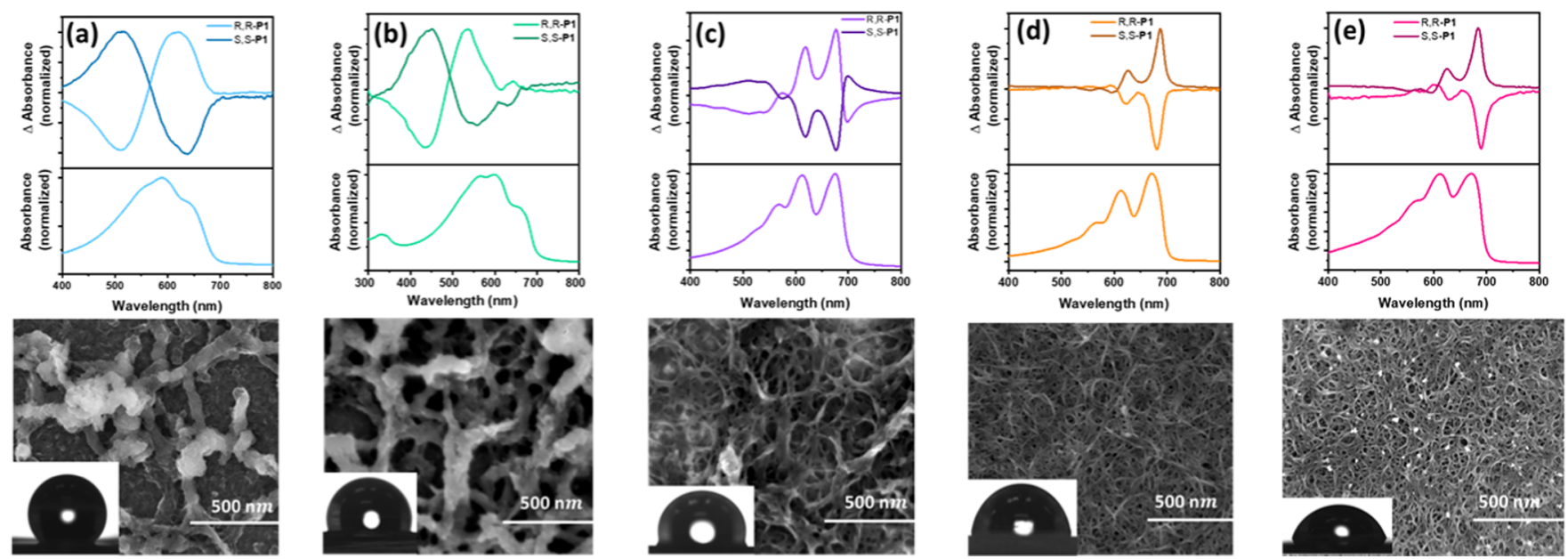

Figure 2. Normalized ECD and UV-vis (upper panels), SEM images (lower panels), and CA measurements (insets) of R,R-P1 and S,S-P1 films synthesized via electropolymerization in acetonitrile with $0.1 \mathrm{M}$ of $(\mathrm{a}) \mathrm{Bu}_{4} \mathrm{NClO}_{4}\left(\mathrm{CA}=125^{\circ}\right),(\mathrm{b}) \mathrm{LiClO}_{4}\left(\mathrm{CA}=104^{\circ}\right),(\mathrm{c}) \mathrm{Bu}_{4} \mathrm{NCF}_{3} \mathrm{SO}_{3}\left(\mathrm{CA}^{\circ}\right.$ $\left.108^{\circ}\right)$, (d) $\mathrm{Bu}_{4} \mathrm{NPF}_{6}\left(\mathrm{CA}=85^{\circ}\right)$, or $(\mathrm{e}) \mathrm{Bu}_{4} \mathrm{NBF}_{4}\left(\mathrm{CA}=72^{\circ}\right)$. 

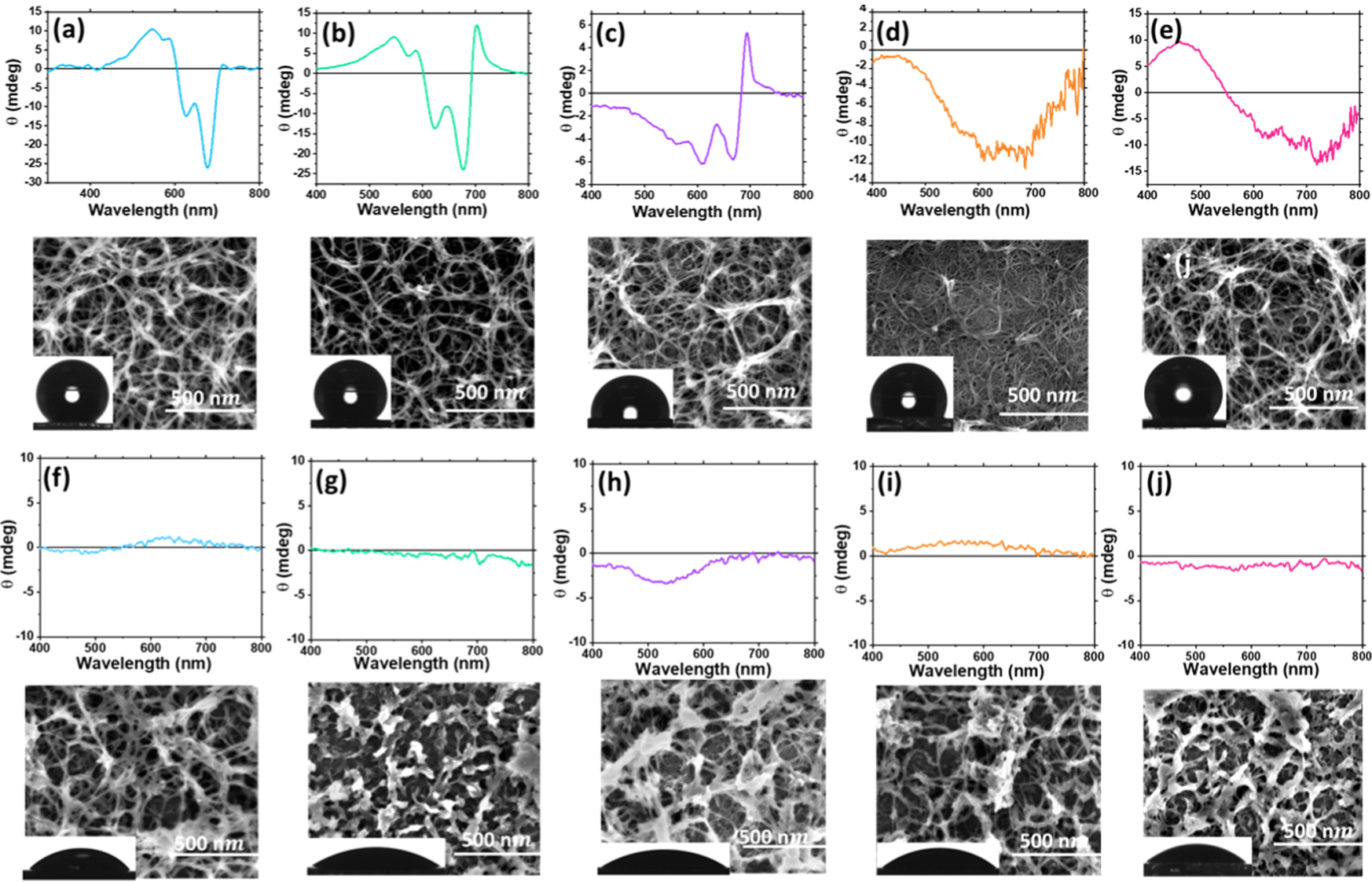

Figure 3. ECD spectra (upper panels) and SEM images (lower panels) and CA measurements (insets) of S,S-P2 films synthesized via electropolymerization in acetonitrile with $0.1 \mathrm{M}(\mathrm{a}) \mathrm{Bu}_{4} \mathrm{NClO}_{4}\left(\mathrm{CA}=150^{\circ}\right),(\mathrm{b}) \mathrm{LiClO}_{4}\left(\mathrm{CA}=146^{\circ}\right),(\mathrm{c}) \mathrm{Bu}_{4} \mathrm{NCF}_{3} \mathrm{SO}_{3}\left(\mathrm{CA}^{\circ} 100^{\circ}\right),(\mathrm{d})$ $\mathrm{Bu}_{4} \mathrm{NPF}_{6}\left(\mathrm{CA}=118^{\circ}\right)$, or $(\mathrm{e}) \mathrm{Bu}_{4} \mathrm{NBF}_{4}\left(\mathrm{CA}=134^{\circ}\right)$ and poly $(3,4$-ethylenedioxythiophene) (PEDOT) films synthesized via electropolymerization in acetonitrile with $0.1 \mathrm{M}(\mathrm{f}) \mathrm{Bu}_{4} \mathrm{NClO}_{4}\left(\mathrm{CA}=45^{\circ}\right),(\mathrm{g}) \mathrm{LiClO}_{4}\left(\mathrm{CA}=22^{\circ}\right),(\mathrm{h}) \mathrm{Bu}_{4} \mathrm{NCF}_{3} \mathrm{SO}_{3}\left(\mathrm{CA}=33^{\circ}\right),(\mathrm{i}) \mathrm{Bu}_{4} \mathrm{NPF}_{6}\left(\mathrm{CA}_{2}=28^{\circ}\right)$, and $(\mathrm{j})$ $\mathrm{Bu}_{4} \mathrm{NBF}_{4}\left(\mathrm{CA}=33^{\circ}\right)$.

chiroptical activity stems from the difference in chirality rather than film anisotropy. In the case of perchlorate anion with either tetrabutylammonium (Figure $2 \mathrm{a}$ ) or lithium (Figure $2 \mathrm{~b}$ ) cations, the SEM images reveal a clusterlike morphology in contrast to the wirelike morphology observed in the case of $\mathrm{Bu}_{4} \mathrm{NBF}_{4}$ and $\mathrm{Bu}_{4} \mathrm{NPF}_{6}$ (Figure 2d,e, respectively). The wirelike morphology of the latter films (Figure 2d,e) also exhibits smaller contact angles (85 and $72^{\circ}$, respectively) compared with the perchlorate-containing films (Figure 2a,b; 125 and $104^{\circ}$, respectively), indicating a lower effective surface area of the hydrophobic film for the latter. ${ }^{27}$ The ECD spectra are also different for each case, with a bisignate peak for films polymerized in $\mathrm{Bu}_{4} \mathrm{NClO}_{4}$ or $\mathrm{LiClO}_{4}$ electrolyte (Figure 2a,b, respectively), implying strong interchain interactions in both. The UV-vis spectra of the perchlorate-containing polymers (Figure 2a,b) are also noteworthy because of broader vibronic shoulders for the $\pi-\pi^{*}$ transition and a hypsochromic shift of $80 \mathrm{~nm}^{28,29}$ Films electropolymerized with trifluoromethylsulfonate-containing ions (Figure 2c) exhibit different structural motifs compared with both perchlorate-containing (Figure 2a,b) and fluorophosphate-containing (Figure 2d,e) polymers; for example, on ECD, a bisignate peak can be observed in Figure $2 c$, but the spectrum also has clear vibronic shoulders.

As ECD signals in solid samples can also arise from anisotropy, ${ }^{13,30}$ the ECD and linear dichroism (LD) spectra were measured with different film orientations (rotated $90^{\circ}$ and flipped $180^{\circ}$ ) for different samples of R,R-P1 (Figure S26, see SI). Film orientation did not affect the ECD signals, which exhibited the same features regardless of orientation. The LD values are small, and therefore should not significantly influence the observed ECD spectra, indicating that the observed ECD signals arise from chiral features in the polymer film.

To account for the effect of phenyl groups, we electropolymerized the dimethyl derivative, $S, S-2$, in various electrolytes to obtain S,S-P2 (Figure 3). A comparison of the ECD spectra and film morphologies shown in Figure 3a-e with those in Figure $2 a-e$ reveals that changing the electrolyte has a significantly smaller effect on the methyl-containing polymer S,S-P2 (Figure 3). The similarities in the ECD spectra for different electrodeposition conditions are also reflected in similar wirelike morphology observed in SEM. Overall, the ECD of S,S-P2 is less intense compared with that of S,S-P1, as reflected by a significantly weaker anisotropy factor $\left(g_{\text {abs }}\right)$ for the former (Figure S42, see SI). This may arise from a lower intramolecular helicity for the less bulky dimethyl groups compared with the diphenyl groups, as was discussed above. In addition, the phenyl-containing polymer can form $\pi-\pi$ interactions in both an intra- and intermolecular fashion, which further affects its morphology.

The morphology of the electrodeposited film is also affected by the solvent. We observed that the characteristics of films P1 and $\mathbf{P 2}$ formed in the presence of propylene carbonate show little to no dependence on the electrolyte, with similar ECD 
spectra, SEM images, and CA obtained under different conditions (see Figures S39 and S40 in SI). Notwithstanding, films of $\mathbf{P 1}$ exhibit larger features and higher surface coverage compared with those of P2 films, which is again a result of stronger interactions in phenyl-substituted chains (Figures S29 and S32, see SI). One explanation for these differences is the lower solubility of the monomer in propylene carbonate.

To understand the role of chiral groups, parent EDOT was polymerized under the same conditions. As can be observed in Figure $3 \mathrm{f}-\mathrm{j}$, no ECD signal can be detected in the case of achiral PEDOT, which serves as an additional indication that the signals observed for P1 (in Figure 2a-e) and P2 (in Figure $3 \mathrm{a}-\mathrm{e}$ ) are not artifacts of film anisotropy but rather result from the chiral side groups. In addition, the effect of electrolyte on film morphology is small, indicating that the substituents play a crucial role in determining the morphology, together with the chosen anion. The contact angle for PEDOT $\left(22-45^{\circ}\right)$ is significantly lower compared with the CA values of the diphenyl- or dimethyl-substituted polymers $\left(72-150^{\circ}\right)$, which correlate with the lower effective surface area of parent PEDOT films.

To better understand the relationship between the observed ECD and polymer film morphology at the molecular level, TDDFT calculations were performed at the B3LYP/6-31G(d) level. Ter-S,S-1 and ter-R,R-1 were taken as representatives of longer polymer chains. Figure $4 a$ presents the ECD spectra for

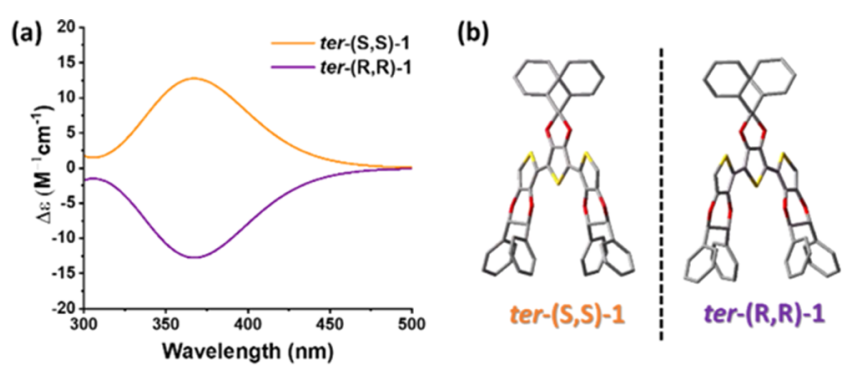

Figure 4. (a) Calculated (TD-DFT/CAM-B3LYP/6-31G(d)) ECD spectra for ter-S,S-1 and ter-R,R-1 and (b) their optimized geometries.

ter-S,S-1 and ter-R,R-1 (Figure 4b), which represent "isolated" polymer chains, displaying a monosignate Cotton effect for the $\pi-\pi^{*}$ transition. This band originates in the intrachain helicity. Repulsion between the phenyl groups produces an interring dihedral angle of $9^{\circ}$. The $\mathrm{P}$ helicity of the $S, S$ enantiomer and the $\mathrm{M}$ helicity of the $R, R$ enantiomer correspond to the positive and the negative signals, respectively. These spectra are consistent with the monosignate peak observed for S,S-P1 polymerized in $0.1 \mathrm{M} \mathrm{Bu}_{4} \mathrm{NPF}_{6}$ or $\mathrm{Bu}_{4} \mathrm{NBF}_{4}$ in acetonitrile (Figure 2d,e, respectively), indicating long intermolecular distances and weak interactions between the polymer chains in these cases.

Next, the ECD signals for dimers of ter-S,S-1 at different orientations were studied to understand their intermolecular interactions. The interchain distance $(r)$ and angles $(\theta)$ were taken as independent variables. ${ }^{31}$ Figure 5 displays the geometry (c) and the calculated ECD spectra (b) for dimers of ter-S,S-1 with $r=7 \AA$ and $\theta=10^{\circ}$. Although the calculated absorption spectra are similar for both the monomer (Figure S43, see SI) and dimer (Figure S45, see SI) of ter-S,S-1, their calculated ECD spectra are substantially different. The calculated ECD spectrum for the dimer of ter-S,S-1 (Figure $5 b)$ is in a good agreement with the experimental spectrum
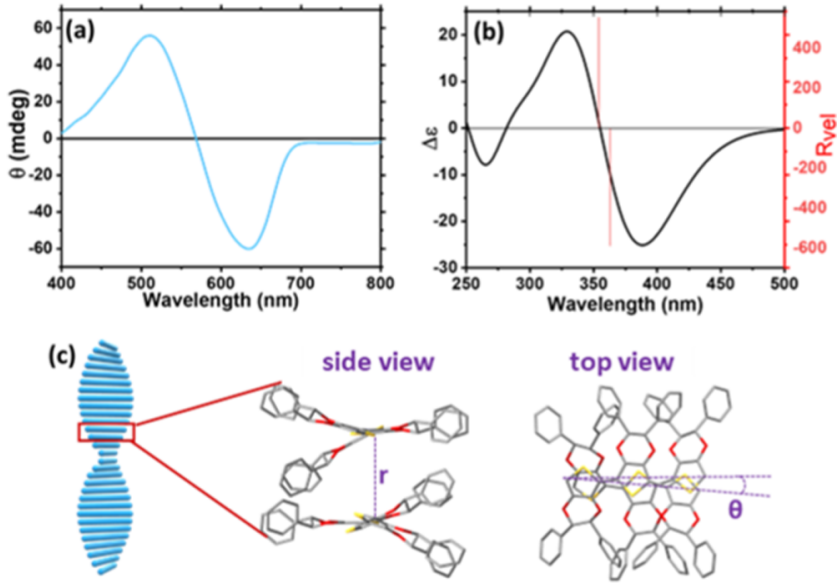

Figure 5. (a) Experimental spectrum of S,S-P1 films synthesized via electropolymerization with $0.1 \mathrm{M} \mathrm{TBPC}$ in acetonitrile. (b) Computational spectrum at the TD-DFT/CAM-B3LYP/6-31G(d) level of the ter-S,S-1 dimer. Both (a) and (b) show a bisignate Cotton effect resulting from interchain interactions. (c) Computational geometries of the ter-S,S-1 dimer, viewed from the top and side, showing close interactions between the chains. The interchain distance and angle are represented by $r$ and $\theta$, respectively.

observed for S,S-P1 synthesized in the presence of perchlorate in acetonitrile (Figure 2a). The observed bisignate peak is negative at higher wavelengths for the $S, S$ enantiomer in both the experimental and computational spectra. This suggests that the observed SEM morphology corresponds to helical intermolecular orientation and/or closer interchain distances, which is reflected in "clusterlike" morphology (Figure 2a,b) compared with the cases in which the ECD spectra lack a bisignate peak, indicating either $\theta$ close to $0^{\circ}$ and/or large interchain distances (isolated chromophores), and a "wirelike" morphology (Figure 2d,e). Therefore, the ECD spectra, SEM images, and CA all reflect various aspects of polymer film morphology, with ECD spectra reflecting the film's molecular characteristics, whereas SEM and CA reflecting its macromolecular characteristics. Consequently, ECD can be applied as an analytical tool for the morphological characterization of chiral conducting polymers.

Meso-1 and Enantiomeric Excess Polymers. To further study the effect of enantiomers vs diastereomers, meso-1 was electropolymerized to yield meso-P1. Whereas meso- 1 shows no optical activity in the ECD spectrum, the polymer, meso-P1, exhibits intense ECD signals. In principle, polymerization of meso monomers should result in a stereo-irregular polymer, which should not show optical activity. However, as can be observed, meso-P1 films that were polymerized with $\mathrm{Bu}_{4} \mathrm{NClO}_{4}$ (Figure 6a) or $\mathrm{Bu}_{4} \mathrm{NCF}_{3} \mathrm{SO}_{3}$ (Figure $6 \mathrm{~b}$ ) electrolytes exhibit strong optical activity. LD experiments as well as ECD measurements at different angles show that this is not the result of film anisotropy but rather arises from a chiral morphology. As the signal always appears with the same sign, we examined the optical rotation of the commercial mesohydrobenzoin used for the synthesis of meso-1 monomer and found it had a small, positive optical rotation $\left(\left[\alpha_{28 / \mathrm{D}}\right]=1.7^{\circ}\right.$ compared with $\left[\alpha_{24 / \mathrm{D}}\right]= \pm 93^{\circ}$ reported for the enantiopure molecule). ${ }^{32}$ The intense ECD signal observed for the polymer indicates an ordered film, which contributed to this amplification. $^{33,34}$ Indeed, in some of the fibers in Figure $6 \mathrm{a}, \mathrm{b}$, a helical motif can be observed. ${ }^{33,34}$ 

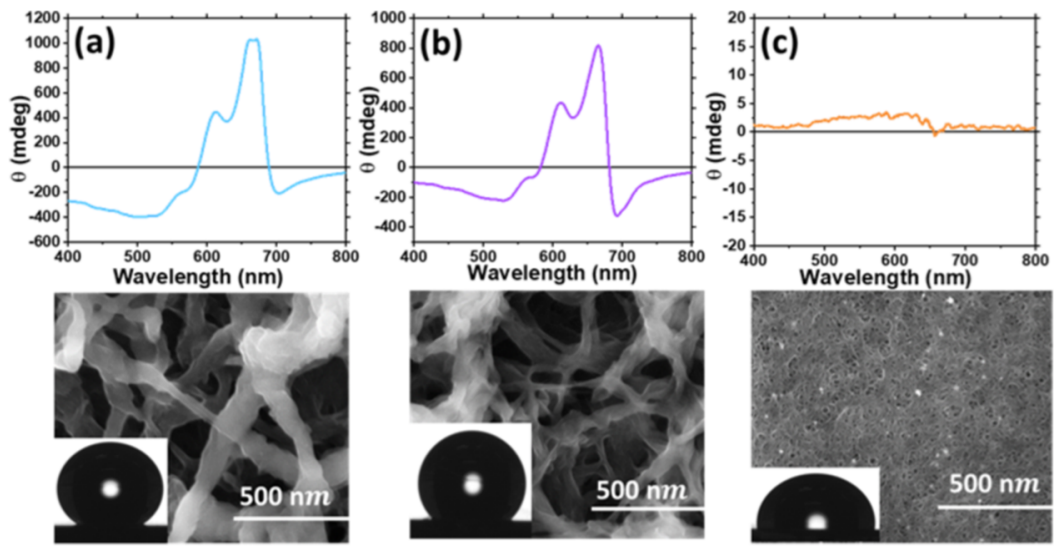

Figure 6. ECD (upper panels), SEM (lower panels), and CA measurements (insets) of meso-P1 synthesized via electropolymerization in acetonitrile and $0.1 \mathrm{M}(\mathrm{a}) \mathrm{Bu}_{4} \mathrm{NClO}_{4}\left(\mathrm{CA}=146^{\circ}\right)$, (b) $\mathrm{Bu}_{4} \mathrm{NCF}_{3} \mathrm{SO}_{3}\left(\mathrm{CA}=97^{\circ}\right)$, or $(\mathrm{c}) \mathrm{Bu}_{4} \mathrm{NPF}_{6}\left(\mathrm{CA}=138^{\circ}\right)$.

One explanation for this observation is chiral amplification, or the "majority rule", which was observed by Meijer's group for chemically polymerized polythiophenes. ${ }^{24}$ To study this phenomenon further, we electropolymerized solutions containing both $S, S-1$ and $R, R-1$ with different enantiomer excesses. As can be seen in Figure 7, even excess of a small
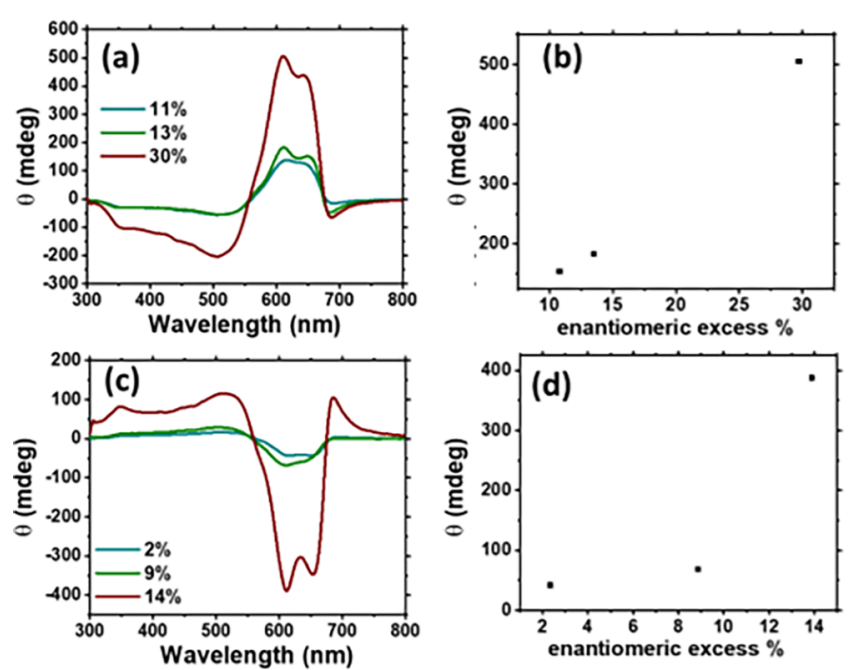

Figure 7. Films polymerized in the presence of various enantiomeric excesses (\%). (a, b) ECD and (c and d) optical activity of polymer films synthesized from enantiomeric mixtures containing an excess of (a, c) $R, R-1$ or (c, d) S,S-1.

( $2 \%$ ) enantiomer, which is hardly observed in the ECD spectra, can direct the overall assembly process and produce polymers with a strong ECD signal, with opposite enantiomers yielding the expected mirror image signals. This finding indicates that chiral amplification, which was previously observed for chemically polymerized films, can also occur during electropolymerization.

\section{CONCLUSIONS}

In this work, we investigated the applicability of ECD as an analytical tool for the characterization of film morphology. We found a direct correlation between the ECD spectra, representing arrangements at the supramolecular level, and the film morphology observed in SEM images. Specifically, exciton coupling, which arises from the supramolecular helical structure and is observed as a bisignate signal in the ECD spectrum, results in polymer films whose SEM images show a clusterlike morphology of larger fibers and whose larger CA values indicate higher effective surface coverage. By contrast, a monosignate ECD signal is associated with a thin-wire morphology and lower effective surface coverage, according to the SEM and CA measurements. The phenyl side chains induce helical arrangements with close interchain interactions and display greater dependence on the electropolymerization conditions compared with methyl side chains. Specifically, the presence of perchlorate ions induces strong exciton coupling. The presence of small enantiomeric excess in either the meso monomer or mixture of both enantiomers results in a strong ECD signal, so providing the first indication of chiral amplification in electropolymerization. Overall, this work demonstrates the power of ECD as an analytical tool to predict the morphology of electropolymerized chiral conducting polymer films.

\section{ASSOCIATED CONTENT}

\section{SI Supporting Information}

The Supporting Information is available free of charge at https://pubs.acs.org/doi/10.1021/acs.macromol.0c01731.

Synthesis, characterization, spectroscopy, and computation.(PDF)

Cartesian coordinates of optimized structures of the geometries presented in the work (TXT)

\section{AUTHOR INFORMATION}

\section{Corresponding Author}

Ori Gidron - The Hebrew University of Jerusalem, 9190401

Jerusalem, Israel; 이이이.org/0000-0002-7037-0563;

Email: ori.gidron@mail.huji.ac.il

\section{Authors}

Dana Amsallem - The Hebrew University of Jerusalem, 9190401 Jerusalem, Israel

Anjan Bedi - The Hebrew University of Jerusalem, 9190401 Jerusalem, Israel; 이이.org/0000-0001-6418-4409

Francesco Tassinari - Department of Chemical and Biological Physics, Weizmann Institute of Science, 76100 Rehovot, Israel

Complete contact information is available at:

https://pubs.acs.org/10.1021/acs.macromol.0c01731 


\section{Funding}

This research was supported by the Israel Science Foundation (grant no. 1789/16). A.B. is supported by a PBC fellowship.

\section{Notes}

The authors declare no competing financial interest.

\section{ACKNOWLEDGMENTS}

We thank the Nano-characterization Unit of the Hebrew University, under the administration of Dr. Shimon Eliav; Dr. Vitaly Gutkin for the SEM operation; the crystallography lab at the Hebrew University of Jerusalem, run by Dr. Benny Bogoslavsky; and Yuval Madmon and Prof. Roie Yerushalmi for assistance with the contact angle measurements.

\section{ABBREVIATIONS}

EDOT, 3,4-ethylenedioxythiophene; 1, diphenyl-EDOT; 2, dimethyl-EDOT; $\mathrm{Bu}_{4} \mathrm{NClO}_{4}$, tetra- $n$-butylammonium perchlorate; $\mathrm{Bu}_{4} \mathrm{NBF}_{4}$, tetra- $n$-butylammonium tetrafluoroborate; $\mathrm{Bu}_{4} \mathrm{NCF}_{3} \mathrm{SO}_{3} \mathrm{Bu}_{4} \mathrm{NPF}_{6}$, tetra- $n$-butylammonium hexafluorophosphate; and $\mathrm{LiClO}_{4}$, lithium perchlorate

\section{REFERENCES}

(1) Kane-Maguire, L. A. P.; Wallace, G. G. Chiral conducting polymers. Chem. Soc. Rev. 2010, 39, 2545-2576.

(2) Brandt, J. R.; Salerno, F.; Fuchter, M. J. The added value of small-molecule chirality in technological applications. Nat. Rev. Chem. 2017, 1, No. 0045.

(3) Sannicolò, F.; Arnaboldi, S.; Benincori, T.; Bonometti, V.; Cirilli, R.; Dunsch, L.; Kutner, W.; Longhi, G.; Mussini, P. R.; Panigati, M.; et al. Potential-driven chirality manifestations and impressive enantioselectivity by inherently chiral electroactive organic films. Angew. Chem., Int. Ed. 2014, 53, 2623-2627.

(4) Mondal, P. C.; Kantor-Uriel, N.; Mathew, S. P.; Tassinari, F.; Fontanesi, C.; Naaman, R. Chiral Conductive Polymers as Spin Filters. Adv. Mater. 2015, 27, 1924-1927.

(5) Sannicolò, F.; Mussini, P. R.; Benincori, T.; Cirilli, R.; Abbate, S.; Arnaboldi, S.; Casolo, S.; Castiglioni, E.; Longhi, G.; Martinazzo, R.; et al. Inherently chiral macrocyclic oligothiophenes: easily accessible electrosensitive cavities with outstanding enantioselection performances. Chem. - Eur. J. 2014, 20, 15298-15302.

(6) Elschner, A.; Kirchmeyer, S.; Lovenich, W.; Merker, U.; Reuter, K. PEDOT: Principles and Applications of an Intrinsically Conductive Polymer; CRC Press, 2010.

(7) Martin, D. C.; Wu, J.; Shaw, C. M.; King, Z.; Spanninga, S. A.; Richardson-Burns, S.; Hendricks, J.; Yang, J. The Morphology of Poly(3,4-Ethylenedioxythiophene). Polym. Rev. 2010, 50, 340-384.

(8) Kirchmeyer, S.; Reuter, K. Scientific importance, properties and growing applications of poly (3, 4-ethylenedioxythiophene). J. Mater. Chem. 2005, 15, 2077-2088.

(9) Groenendaal, L.; Jonas, F.; Freitag, D.; Pielartzik, H.; Reynolds, J. R. Poly (3, 4-ethylenedioxythiophene) and its derivatives: past, present, and future. Adv. Mater. 2000, 12, 481-494.

(10) Verswyvel, M.; Koeckelberghs, G. Chirality in conjugated polymers: when two components meet. Polym. Chem. 2012, 3, 32033216.

(11) Wang, P.; Jeon, I.; Lin, Z.; Peeks, M. D.; Savagatrup, S.; Kooi, S. E.; Van Voorhis, T.; Swager, T. M. Insights into Magneto-Optics of Helical Conjugated Polymers. J. Am. Chem. Soc. 2018, 140, 65016508.

(12) Jeong, Y. S.; Akagi, K. Control of Chirality and Electrochromism in Copolymer-Type Chiral PEDOT Derivatives by Means of Electrochemical Oxidation and Reduction. Macromolecules 2011, 44, 2418-2426.

(13) Matsushita, S.; Jeong, Y. S.; Akagi, K. Electrochromism-driven linearly and circularly polarised dichroism of poly (3, 4-ethyl- enedioxythiophene) derivatives with chirality and liquid crystallinity. Chem. Commun. 2013, 49, 1883-1890.

(14) Goto, H. Circular Dichroism of Bipolarons in a Chiroptically Active Conjugated Polymer. J. Macromol. Sci., Part B 2016, 55, 471482.

(15) Caras-Quintero, D.; Bäuerle, P. Synthesis of the first enantiomerically pure and chiral, disubstituted 3, 4-ethylenedioxythiophenes (EDOTs) and corresponding stereo-and regioregular PEDOTs. Chem. Commun. 2004, 926-927.

(16) Darmanin, T.; Klimareva, E. L.; Schewtschenko, I.; Guittard, F.; Perepichka, I. F. Exceptionally Strong Effect of Small Structural Variations in Functionalized 3,4-Phenylenedioxythiophenes on the Surface Nanostructure and Parahydrophobic Properties of Their Electropolymerized Films. Macromolecules 2019, 52, 8088-8102.

(17) Bellanger, H.; Darmanin, T.; Taffin de Givenchy, E.; Guittard, F. Chemical and Physical Pathways for the Preparation of Superoleophobic Surfaces and Related Wetting Theories. Chem. Rev. 2014, $114,2694-2716$

(18) del Valle, M. A.; Ramírez, A.; Hernández, L.; Armijo, F.; Díaz, F.; Arteaga, G. Influence of the supporting electrolyte on the electrochemical polymerization of 3, 4-ethylenedioxythiophene. Effect on $\mathrm{p}$-and $\mathrm{n}$-doping/undoping, conductivity and morphology. Int. J. Electrochem. Sci. 2016, 11, 7048-7065.

(19) Seki, Y.; Takahashi, M.; Takashiri, M. Effects of different electrolytes and film thicknesses on structural and thermoelectric properties of electropolymerized poly (3, 4-ethylenedioxythiophene) films. RSC Adv. 2019, 9, 15957-15965.

(20) Louet, C.; Cantin, S.; Dudon, J.-P.; Aubert, P.-H.; Vidal, F.; Chevrot, C. A comprehensive study of infrared reflectivity of poly (3, 4-ethylenedioxythiophene) model layers with different morphologies and conductivities. Sol. Energy Mater. Sol. Cells 2015, 143, 141-151.

(21) Ming, S.; Feng, Z.; Mo, D.; Wang, Z.; Lin, K.; Lu, B.; Xu, J. Solvent effects on electrosynthesis, morphological and electrochromic properties of a nitrogen analog of PEDOT. Phys. Chem. Chem. Phys. 2016, 18, 5129-5138.

(22) Patra, S.; Barai, K.; Munichandraiah, N. Scanning electron microscopy studies of PEDOT prepared by various electrochemical routes. Synth. Met. 2008, 158, 430-435.

(23) Poverenov, E.; Li, M.; Bitler, A.; Bendikov, M. Major Effect of Electropolymerization Solvent on Morphology and Electrochromic Properties of PEDOT Films. Chem. Mater. 2010, 22, 4019-4025.

(24) Langeveld-Voss, B.; Waterval, R.; Janssen, R.; Meijer, E. Principles of "majority rules" and "sergeants and soldiers" applied to the aggregation of optically active polythiophenes: Evidence for a multichain phenomenon. Macromolecules 1999, 32, 227-230.

(25) Kaloni, T. P.; Giesbrecht, P. K.; Schreckenbach, G.; Freund, M. S. Polythiophene: From Fundamental Perspectives to Applications. Chem. Mater. 2017, 29, 10248-10283.

(26) Taniguchi, T.; Usuki, T. Circular Dichroism Spectroscopy. In Supramolecular Chemistry: From Molecules to Nanomaterials; Wiley, 2012, 393-410. DOI: 10.1002/9780470661345.smc029.

(27) Kwok, D. Y.; Neumann, A. W. Contact angle measurement and contact angle interpretation. Adv. Colloid Interface Sci. 1999, 81, 167249.

(28) Spano, F. C.; Silva, C. H-and J-aggregate behavior in polymeric semiconductors. Annu. Rev. Phys. Chem. 2014, 65, 477-500.

(29) Spano, F. C.; Meskers, S. C.; Hennebicq, E.; Beljonne, D. Probing excitation delocalization in supramolecular chiral stacks by means of circularly polarized light: experiment and modeling. J. Am. Chem. Soc. 2007, 129, 7044-7054.

(30) Nakano, T. Synthesis, structure and function of $\pi$-stacked polymers. Polym. J. 2010, 42, 103-123.

(31) Padula, D.; Santoro, F.; Pescitelli, G. A simple dimeric model accounts for the vibronic ECD spectra of chiral polythiophenes in their aggregated states. RSC Adv. 2016, 6, 37938-37943.

(32) Wang, Z.-M.; Sharpless, K. B. A solid-to-solid asymmetric dihydroxylation procedure for kilogram-scale preparation of enantiopure hydrobenzoin. J. Org. Chem. 1994, 59, 8302-8303. 
(33) Bredas, J. L.; Street, G. B. Polarons, bipolarons, and solitons in conducting polymers. Acc. Chem. Res. 1985, 18, 309-315.

(34) Goto, H. Optical rotation and electron spin resonance of an electro-optically active polythiophene. Mater. Chem. Phys. 2010, 122, 69-72. 